\title{
Monitor Watchers and Alarm Fatigue: Cautious Optimism
}

\author{
Halley Ruppel, MS, RN, CCRN ${ }^{* *}$, Marjorie Funk, PhD, RN, FAHA, FAAN ${ }^{2}$
}

${ }^{1}$ PhD Candidate, Yale School of Nursing, West Haven, Connecticut; ${ }^{2}$ Helen Porter Jayne and Martha Prosser Jayne Professor of Nursing, Yale School of Nursing, West Haven, Connecticut.

Monitor watcher personnel are frequently used to assist nurses with identifying meaningful events on telemetry monitors. Although effectiveness of monitor watchers on patient outcomes has not been demonstrated conclusive$1 y,{ }^{1}$ as many as $60 \%$ of United States hospitals may be using monitor watchers in some capacity. ${ }^{2}$ Presumed benefits of monitor watchers include prompt recognition of changes in patients' conditions and the potential to reduce alarm fatigue among hospital staff. Alarm fatigue is desensitization resulting from overexposure to alarm signals that are either invalid or clinically irrelevant. Alarm fatigue has resulted in missed patient events and preventable deaths. ${ }^{3}$ In this issue of the Journal of Hospital Medicine, Palchaudhuri et al. ${ }^{4}$ report findings from their observational study of telemetry monitor alarms intercepted by monitor watchers as a mechanism for reducing both nurses' exposure to alarm signals and subsequent alarm fatigue.

To our knowledge, the study by Palchaudhuri et al. ${ }^{4}$ is the first to report the effect of monitor watchers on nurses' exposure to alarm signals. In this study, over a 2-month period monitor watchers intercepted $87 \%$ of alarms before they were sent to the nurse's telephone. Monitor watchers intercepted over $90 \%$ of bradycardia and tachycardia alarms, indicating that they believed these alarms to be clinically irrelevant. Monitor watchers also intercepted about $75 \%$ of alarms for lethal arrhythmias, indicating that they believed these alarms to be invalid.

In this study, decisions about alarm validity and relevance were made through close communication between monitor watchers and nursing staff. If an alarm was sounding and the monitor watcher had already spoken with the nurse about it and established that the nurse was addressing the problem, the monitor watcher would intercept subsequent alarms for that issue or event (according to personal communication with S. Palchaudhuri). The results of the study not only indicate that monitor watchers can reduce the number of alarms to which a nurse is exposed, but also support previous findings that few alarms are valid or clinically relevant..$^{5-7}$ The results of this study also suggest that "nuisance" alarms should include not only clinically irrelevant alarms, but also relevant alarms for which the nurse is actively seeking a

\footnotetext{
*Address for correspondence and reprint requests: Halley Ruppel, MS, RN, CCRN, PO Box 27399, West Haven, CT 06516; Telephone: 617-447-6160; Fax: 203-737-4480; E-mail: halley.ruppel@yale.edu
}

Received: January 8, 2017; Accepted: January 12, 2017

2017 Society of Hospital Medicine DOI 10.12788/jhm.2758 solution. Monitor watchers may have an important role in addressing these alarms.

The study raises important considerations regarding monitor watcher practice and alarm fatigue. If monitor watchers are to be effective in reducing nurses' exposure to alarms, they must use good judgment to determine when to intercept an alarm, call the nurse, or both. In the absence of proper judgment, monitor watchers may inadvertently increase nurses' fatigue through redundant calls or inappropriately suppress valid relevant alarms. In free-text responses to our national monitor watcher survey, nurses expressed frustration over redundant calls from monitor watchers for invalid and irrelevant alarms. ${ }^{2}$ Research suggests that monitor watchers may not identify potentially dangerous alarms with complete accuracy. In a recent study reported in The Journal of the American Medical Society (JAMA), monitor watchers missed about $18 \%$ of patients with detectable rhythm or rate changes on telemetry in the hour before an emergency response team was activated. ${ }^{8}$

Several factors and conditions may affect monitor watchers' judgment: 1) education and training, 2) location and access to contextual patient information, and 3) fatigue. First, across the US, the level of education required for monitor watcher positions ranges from a high school diploma to licensure as a registered nurse. The content and frequency of in-service training required also varies. ${ }^{2}$ These differing requirements may influence monitor watchers' ability to interpret alarms.

Second, most monitor watchers are located off the patient care unit, ${ }^{2}$ which influences their access to information. Even in remote locations, monitor watchers can assess alarm validity by reviewing parameter waveforms for artifact. However, determining the relevance of an alarm to a particular patient is a more complex task requiring contextual information about the patient. ${ }^{9}$ Monitor watchers must work closely with clinicians at the bedside to determine the relevance of alarms, and repeated contact between monitor watchers and nurses over alarm conditions may itself increase nurses' alarm fatigue.

Finally, fatigue may affect monitor watchers themselves and reduce their effectiveness. This issue was raised by $\mathrm{Pal}$ chaudhuri et al. Both the number of monitors watched and the length of the monitor watcher's shift likely influence alertness and effectiveness. In a simulation study, Segall et al. ${ }^{10}$ found that monitor watchers' recognition of serious arrhythmias was significantly delayed when they were responsible for more than 40 patient monitors. Monitor watchers often work 12-hour shifts, ${ }^{2}$ and although no research has 
been reported on their shift-related alertness, this is a long time to remain attentive.

Given these potential challenges, future research should specifically address adverse patient outcomes and missed clinically relevant alarms. Only two of the seven patients who arrested during the study by Palchaudhuri et al. ${ }^{4}$ were on telemetry, and neither arrested due to lethal arrhythmias. While this is an important indication that no alarms for lethal arrhythmias were inadvertently suppressed, it is difficult to achieve adequate statistical power to assess rare outcomes like cardiac arrests. In a future study, alarms intercepted by monitor watchers could be assessed for accuracy and relevance to patient care to determine whether important alarms were inadvertently suppressed.

In summary, the study by Palchaudhuri et al. ${ }^{4}$ represents a preliminary step in considering the potential utility of monitor watchers for reducing invalid and clinically irrelevant alarms as well as subsequent alarm fatigue. As the authors note, dedicated monitor watchers can screen alarms much more quickly than nurses who may be engaged in other activities when an alarm signals. The study raises interesting questions about how monitor watchers should be incorporated into workflow. Should their only responsibility be to call regarding potentially critical events, or should they be able to prevent alarms from reaching the nurse? Could monitor watchers provide guidance to reduce alarm fatigue, such as suggesting parameter changes when they see trends in irrelevant alarms? Future research is warranted to understand how monitor watchers can be used most effectively to reduce alarm fatigue, and which characteristics of monitor watchers and their practice result in the best patient outcomes.

Disclosure: Nothing to report.

\section{References}

1. Funk M, Parkosewich JA, Johnson CR, Stukshis I. Effect of dedicated monitor watchers on patients' outcomes. Am J Crit Care. 1997;6(4):318-323.

2. Funk M, Ruppel H, Blake N, Phillips J. Research: Use of monitor watchers in hospitals: characteristics, training, and practices. Biomed Instrum Technol. 2016;50(6):428-438.

3. Joint Commission. Medical device alarm safety in hospitals. Sentinel Event Alert. 2013;(50):1-3.

4. Palchaudhuri S, Chen S, Clayton E, Accurso A, Zakaria S. Telemetry monitor watchers reduce bedside nurses' exposure to alarms by intercepting a high number of nonactionable alarms. J Hosp Med. 2017;12(6):447-449.

5. Bonafide CP, Lin R, Zander M, et al. Association between exposure to nonactionable physiologic monitor alarms and response time in a children's hospital. J Hosp Med. 2015;10(6):345-351.

6. Drew BJ, Harris P, Zègre-Hemsey JK, et al. Insights into the problem of alarm fatigue with physiologic monitor devices: a comprehensive observational study of consecutive intensive care unit patients. PLoS One. 2014;9(10):e110274.

7. Siebig S, Kuhls S, Imhoff M, Gather U, Schölmerich J, Wrede CE. Intensive care unit alarms-how many do we need? Crit Care Med. 2010;38(2):451-456.

8. Cantillon DJ, Loy M, Burkle A, et al. Association between off-site central monitoring using standardized cardiac telemetry and clinical outcomes among non-critically ill patients. JAMA. 2016;316(5):519-524.

9. Rayo MF, Moffatt-Bruce SD. Alarm system management: evidence-based guidance encouraging direct measurement of informativeness to improve alarm response. BMJ Qual Saf. 2015;24(4):282-286.

10. Segall N, Hobbs G, Granger CB, et al. Patient load effects on response time to critical arrhythmias in cardiac telemetry: a randomized trial. Crit Care Med. 2015;43(5):1036-1042. 